\title{
ENQUETES ÉPIDÊMIOLOGIQUES
}

\section{SUR LA MALADIE DE C. CHAGAS AU MEXIQUE (1). RÉDUVIDÉS VECTEURS. ANIMAUX RÉSERVOIRS DF, VIRUS. CAS HUMAINS}

\author{
Par E. BRUMPT, Luis MAZZOTI et L. C. BRUMPT (2)
}

Nous donnons, dans ce travail, un court exposé des enquêtes poursuivies par nous, depuis 1932, dans diverses régions du Mexique, où la trypanosomose américaine semblait ne pas exister. Des recherches récentes, que nous allons résumer, montrent que la maladie, bien que bénigne, est probablement assez répandue.

Nous étudierons successivement les réduvidés hématophages, hôtes intermédiaires du Trypanosoma cruzi, les animaux réservoirs de virus, enfin, les cas humains reconnus depuis quelques mois seulement.

\section{Réduvidés vecteurs du Trypanosoma cruzi au Mexigue}

Trypanosoma cruzi, découvert par C. Chagas en 1909 dans le tube digestif de Triatoma megista, est certainement le trypanosome le plus indifférent en ce qui concerne, d'une part, le mammifère chez lequel il accomplit une partie de son cycle et, d'autre part, l'invertébré chez lequel il termine son évolution. Dès 1912 en effet, l'un de nous (E. B.) a établi que ce trypanosome pouvait donner des formes métacycliques infectieuses chez diverses punaises : Cimex lectularius, $C$. rotundatus, $C$. boueti et $C$. hirundinis, ainsi que chez un acarien, l'Ornithodorus moubata. Ces recherches ont été confirmées

(1) Nous adressons nos bien vifs remerciements à M. le Docteur Andreu Almazan qui, en 1932, alors qu'il était Gouverneur de l'Etat de Puebla, puis en 1938, comme Ministre de la Santé Publique, nous a donné toutes les facilités pour effectuer diverses enquêtes épidémiologiques dans plusieurs états de la République mexicaine.

(2) En ce qui concerne la partie des recherches effectuées à Paris, nous exprimons notre sincère gratitude, pour leur précieuse collaboration technique, au $\mathrm{D}^{\mathrm{r}}$ Annette Herr qui a contracté accidentellement, à la fin de décembre 1938, la maladie de Chagas dont elle est heureusement guérie, et à Mlle A. Buttner, qui a assuré le service de sa compagne pendant la durée de la maladie de cette dernière.

Annales de Parastrologie, T. XVII, $\mathrm{N}^{\circ} 4 .-1^{\text {er }}$ juillet 1939 , p. 299-312. 
par divers auteurs, et, tout récemment, Rodhain et Brussaert (1933) ont même obtenu l'évolution complète chez un diptère pupipare, parasite du mouton, le Melophagus ovinus.

Plus récemment, E. Brumpt (1939) a réussi à infecter avec $T$. cruzi, deux autres espèces de punaises, l'une parasite des hirondelles du Mexique et différente de Cimex hirundinis, l'autre parasite des chauves-souris d'Allemagne (Cimex stadleri), ainsi qu'un certain nombre d'ornithodores (O. coniceps, O. lahorensis, O. migonei, O. nicollei, $O$. rostratus, O. tholozani (= papillipes), O. savignyi, O. turicata, O. venezuelensis). Hoare (1938) a obtenu une évolution complète, dans 100 p. 100 des cas, dans la cavité générale de chenilles de Galleria mellonella.

Cette grande ubiquité parasitaire permet de comprendre pourquoi l'évolution du Trypanosoma cruzi a toujours été obtenue expérimentalement cbez tous les réduvidés hématophages étudiés, nourris sur des mammifères infectés.

On sait actuellement que sur les 70 espèces de réduvidés hématophages (1) habitant l'Amérique, 26 ont été trouvées infectées dans.la nature et 4 ont pu l'être expérimentalement. C'est dire que l'évolution doit se poursuivre chez toutes les espèces connues.

Onze espèces de triatomes et une espèce de Rhodnius ont été rencontrées au Mexique. Ce sont, par ordre alphabétique : Triatoma barberi Usinger, 1939 ; T. dimidiata (Latreille, 1811) ; T. gerstaeckeri (Stål, 1859) ; T. maculipennis (Stål, 1859) ; T. (E.) maxima (Uhler, 1894) ; T. mexicana (H. Schaeffer, 1848) ; T. pallidipennis (Stål, 1872) ; T. phyllosoma (Burmeister, 1835) ; T. protracta (Uhler, 1894) ; T. rubida (Uhler, 1894) ; T. (E.) sonoriana (Del Ponte, $1930)$; Rhodnius prolixus Stål, 1859.

Il existe de plus, au sud des Etats-Unis, Triatoma indictiva, $T$. neotoma, T. sanguisuga et T. uhleri qui se rencontreront probablement un jour au Nord du Mexique, quand des recherches entomologiques systématiques seront entreprises.

Au cours des enquêtes que nous avons effectuées, nous avons récolté six espèces seulement de réduvidés, mais parfois en grande abondance. Ce sont $T$. barberi, $T$. dimidiata et sa forme maculipennis ; $T$. pallidipennis ; $T$. phyllosoma à ailes courtes, moyennes et longues; $T$. rubida et Rhodnius prolixus. Toutes ces espèces ont été rencontrées dans des maisons ou dans leurs dépen-

(1) A ces différentes espèces il convient d'ajouter un hémiptère hématophage de petite taille, Clerada apicicorris, appartenant à une famille différente, frouvé dans les chaumières de l'Amazonie (Brésil), par L. de Castro Ferreira et L. Deane (1938) et que ces auteurs ont pu infecter expérimentalement par Trypanosoma cruzi. 
dances; en outre, $T$. pallidipennis a été trouvé en grand nombre, à tous les stades de son évolution, dans des terriers de tatous et surtout d'un gros rat sauvage, Neotoma (Iodomys) alleni, fréquent aux environs de Colima (Col.), et sur lequel se nourrit également dans la nature l'Ornithodorus nicollei qui n'était connu jusqu'ici qu'à l'état domestique, dans les maisons de la région de Mescala (Guerrero).

Nous avons cherché en vain des triatomes dans les longs nids de " calandria » (pl. XXII, fig. 4) suspendus aux arbres des régions chaudes du Mexique et dans lesquels on a trouvé, en République Argentine, certaines espèces telles que Triatoma rugulosa et Triatoma (Psammolestes) coreodes (Mazza), et au Venezuela, T. (Psammolestes) arthuri (J.F. Torrealba, 1937).

Nous n'avons découvert que des scorpions dans les nids de pics creusés dans les trones des grandes cactées candélabres (pl. XXIII, fig. 5).

Pour effectuer des enquêtes fructueuses, concernant l'existence et la fréquence des réduvidés qui transmettent la maladie de Chagas, il est indispensable de montrer aux habitants des villages des échantillons vivants ou morts des triatomes et des Rhodnius que l'on recherche. $\mathrm{Si}$, à la vue de ces échantillons, les gens affirment que ces animaux sentent mauvais et qu'ils se trouvent en abondance sur les arbres, c'est assurément qu'ils ne les connaissent pas et les confondent avec les réduvidés prédateurs. $\mathrm{Si}$, au contraire, leur existence leur est familière, ils savent que ces insectes piquent la nuit et se nourrissent du sang de l'homme et des animaux élevés par lui. Il suffit alors, en général, d'offrir une petite prime pour chaque exemplaire récolté pour en obtenir souvent un nombre considérable.

Cependant, quand ces insectes sont rares et que les habitants des chaumières sont peu curieux, ils ignorent de bonne foi la présence de ces animaux. Dans certains cas, une fausse retenue incite certains d'entre eux à déclarer qu'il n'y a chez eux, ni même dans leur village, aucun de ces insectes qui abondent parfois dans leurs habitations. Leur réponse est aussi humaine, d'ailleurs, que celle des hôteliers qui affirment n'avoir jamais vu de punaises dans des chambres où elles pullulent et qu'ils louent aux voyageurs de passage.

Le tableau ci-joint donne les résultats des études faites par nous depuis 1932 ; il permet de constater que l'infection est aussi intense chez les triatomes récoltés dans des terriers situés très loin des habitations humaines, que chez ceux récoltés dans ces dernières.

La dernière colonne du tableau indique les infections positives obtenues par inoculation d'animaux divers. Signalons cependant 


\section{Fréquence de l'infection des Triatomidés(1) du Mexique par le Trypanosoma cruzi}

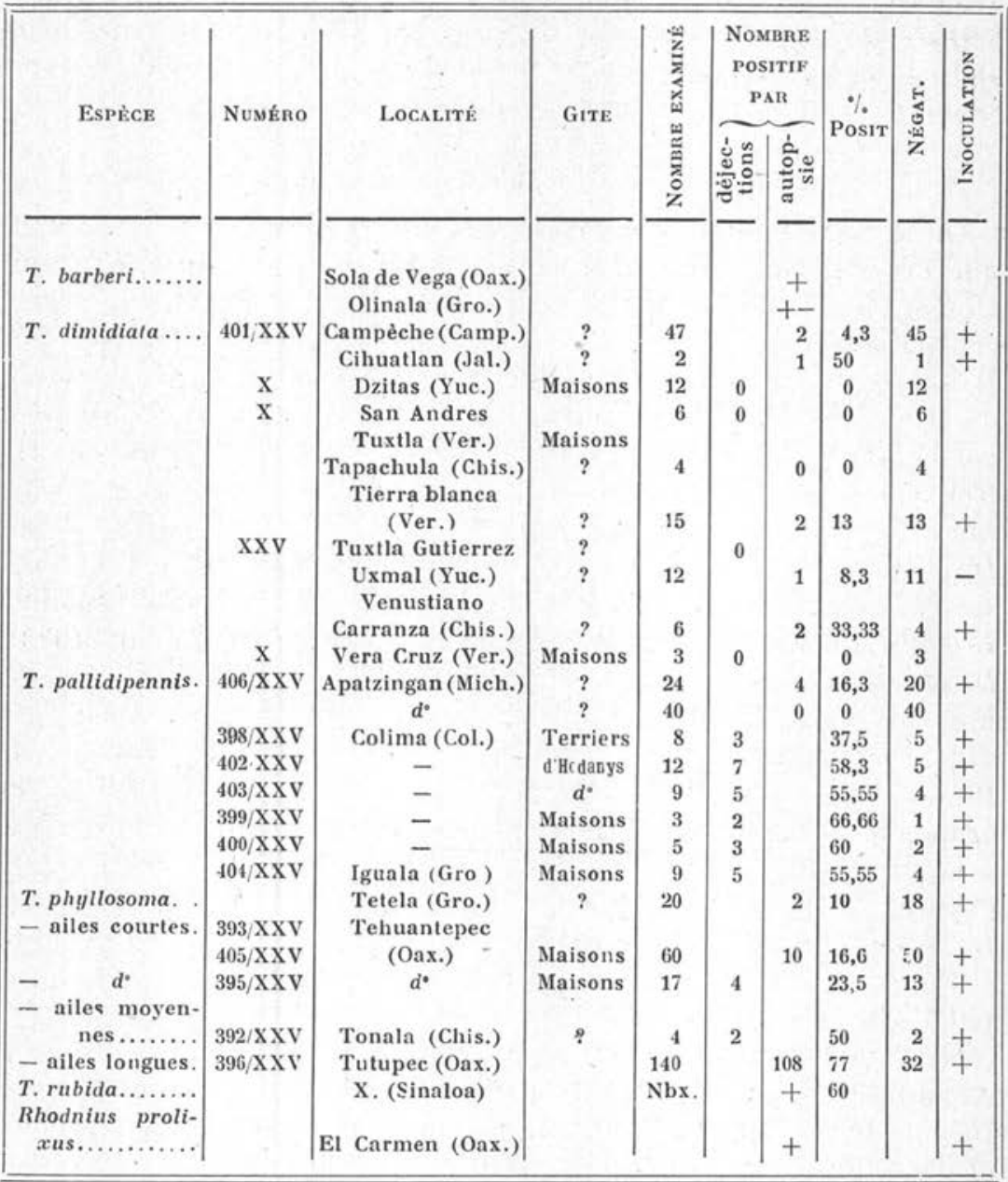

que, dans le cas des Rhodnius prolixus d'El Carmen, où le pourcentage d'insectes présentant des flagellés dans leur intestin atteint 60 , l'un de nous (L. M.) a enregistré un certain nombre d'insuccès, dus peut-être à l'existence d'une autre espèce de flagellé. Nous savons, en effet qu'au Venezuela $\mathrm{S}$. Tejera a décrit, chez ce même

(1) Suivant les localités, ces insectes portent les noms sutvants ; Chincha voladora, Chincha ocicona et Chinchona. 
réduvidé, le Trypanosoma rangeli, non inoculable aux animaux de laboratoire, et que, d'autre part, Rodhain (1937) a réussi à faire évoluer Trypanosoma minasense d'un singe américain (Chrysothrix sciurea), non inoculable à la souris et au singe Macacus rhesus, chez Triatoma megista.

\section{RÉSERVOIRS DE virus}

On sait, depuis les recherches historiques de C. Chagas au Brésil, que Trypanosoma cruzi peut se rencontrer dans la nature, non seulement chez l'homme, mais encore chez divers animaux tels que le chat et surtout certaines espèces de tatous : Dasypus novemcinctus, $D$. sexcinctus et $D$. unicinctus qui sont infectés dans la proportion de 40 p. 100 et auxquels la maladie est transmise par les Triatoma geniculata qui vivent dans leurs terriers. D'autres tatous ont été trouvés porteurs de Trypanosoma cruzi, en particulier Chaetophractus vellerosus, Dasypus hybridus, d'après Mazza, D. paraguayense, D. pentadactylus (?) au Vénézuéla (J.F. Torrealba, 1937). Diverses sarigues sont souvent parasitées : Didelphis aurita (Tejera), D. marsupialis (Robertson, 1929), D. virginana (Kofoid et Donat, 1933), D. paraguayensis et Marmosa mitis. Cette dernière espèce, qui est une sarigue naine, de la taille d'un jeune rat, vit parfois dans les nids d'oiseaux où elle a été trouvée infectée, au Paraguay, par Pifano, cité par Mazza (1939). Les jeunes chiens hébergent également $T$. cruzi en Argentine (Mazza), au Guatemala (Reichenow, 1934) ainsi que les chats au Brésil (C. Chagas) et en Uruguay (Talice) ; il en est de même d'un petit singe de l'Amazone, Chrysothrix sciureus (Chagas, Horta).

Une loutre, Lutreolina crassicauda et plusieurs espèces de mustélidés du genre Grisonella ont été trouvées infectées en Argentine (Mazza, 1937). Un furet, Tayra barbara est réservoir de virus en Argentine (S. Mazza, 1939) et au Brésil (de Castro Ferreira et Deane, 1938), ainsi qu'une belette non déterminée en Uruguay (Talice), cité par Mazza 1939.

Enfin, parmi les rongeurs, citons Neotoma fuscipes de Californie (Kofoid et Mac Culloch, 1916; Kofoid et Donat, 1933) et le surmulot (Mus norvegicus) dont l'infection a été observée une fois au Vénézuéla (J.-F. Torrealba, 1937).

Parmi les animaux domestiques réservoirs de virus, le chat et le chien ont un rôle bien établi, car ils présentent tous deux l'infection naturelle. La réceptivité du mouton a été constatée par E. Brumpt (1913), qui a infecté un agneau (631/I) de trois semai- 
nes en plaçant sur la muqueuse buccale un broyat de triatomes. Au cours de ce présent travail, un jeune porc (500/XXVI), ayant reçu à Paris des déjections de triatomes du Mexique dans l'œil droit, ne montra pas de parasites à l'examen direct du sang, mais un xénodiagnostic effectué un mois après le début de l'expérience se montra positif. Il est donc permis d'ajouter que le porc, très abondant dans les habitations rurales de l'Amérique latine, peut être un réservoir de virus naturel, puisqu'il peut être infecté par les trypanosomes métacycliques des triatomes, dont on connaît le grand pouvoir de pénétration à travers toutes les muqueuses. Le porcelet dont il est parlé ci-dessus ne présenta aucune infection oculaire particulière.

Au Mexique, au cours d'une enquête épidémiologique en 1936, l'un de nous (L. M.) a découvert pour la première fois Trypanosoma cruzi chez divers triatomes, ainsi que chez un chien, alors qu'il enregistrait des résultats négatifs à l'examen direct de trois tatous, dix jeunes chiens, six chats et douze chevreaux.

En 1938, nous avons trouvé une infection spontanée chez un tatou (Dasypus novemcinctus mexicanus) de la région de Colima et, quelques semaines plus tard, un nouveau tatou infecté fut trouvé dans cette même région par le $\mathrm{D}^{r}$ Jesus Calderon (1) par utilisation du xénodiagnostic. Le virus du premier tatou a été conservé sur des triatomes neufs et a permis de donner à Paris de nombreuses infections à des souris blanches.

Un rongeur voisin du Neotoma fuscipes de Californie, Neotoma (Hodomys) alleni (2), abondant à Colima (Mexique), doit certainement être un réservoir de virus efficace, car nous avons trouvé 37,5 à 55,5, p. 100 de Triatoma pallidipennis infectés d'une souche de T. cruzi transmissible à l'homme ; en effet, comme nous le verrons plus loin, une assistante de l'Institut de Parasitologie de Paris a contracté accidentellement une infection aiguë en manipulant cette souche.

Cependant l'étude anatomo-pathologique du cœur de trois rongeurs adultes (pl. XXIV, fig. 8), tués à Colima, s'est montré négative; ce qui peut être dû à une longévité faible des parasites chez ce rongeur contrairement à ce qui s'observe dans le cas des tatous et des

(1) Communication personnelle.

(2) Ce rongeur, qui porte à Colima le nom vulgaire de « comadreja », nom donné d'autre part en Argentine à un putois, vit dans des terriers creusés dans la terre entre les racines des grands figuiers (pl. XXI, fig. 2) ou au pied de falaises calcaires au bord des ruisseaux. Sa présence est facile à déceler par ses déjections qui ressemblent à celles des cobayes et forment des amas ou «escurados », situés toujours à quelque distance de son terrier. 
sarigues qui conservent longtemps des trypanosomes dans le sang périphérique et permettent ainsi de déceler par simple examen direct la fréquence de leur infection.

Nous n'avons pas eu l'occasion d'examiner le sang d'autres animaux mais il est probable que tous les animaux qui s'abritent dans des terriers faits par eux ou qui utilisent ceux des autres, doivent présenter des infections qui pourront être mises en évidence, soit par l'examen direct, soit par le xénodiagnostic si les parasites n'apparaissent pas dans le sang. Les plus suspects sont le coyote (Canis latrans), les spermophiles (Citellus annulatus annulatus) et le «tesmo », les sarigues, les coatis, etc. Nous devons cependant signaler que deux jeunes coatis, que nous avons inoculés à Colima avec le sang d'un tatou montrant de rares parasites ne se sont pas infectés, ainsi qu'il résulte d'examens directs et d'un xénodiagnostic demeurés négatifs.

\section{Cas humains déterminés PAR LES SOUChes}

\section{DE TRYPANOSOMA CRUZI MEXICAINES}

La présence de nombreux réduvidés hématophages infectés dans la nature, même lorsque ces animaux se sont adaptés à l'habitation humaine, ne permet pas d'affirmer l'existence de l'infection humaine. C'est ainsi que la maladie de Chagas n'a pas encore été signalée aux Etats-Unis bien que des Triatoma protracta et des T. uhleri, récoltés dans des habitations, aient été trouvés infectés, les premiers en Californie (Kofoid et Donat, 1933), les seconds en Arizona (Kofoid et Whitaker, 1936).

Les nombreux triatomes signalés porteurs de $T$. cruzi au Mexique ne permettaient pas d'affirmer l'existence de l'infection humaine. En effet, les enquêtes de 1932, 1936 et 1938 portant sur la recherche des signes cliniques, de l'œdème facial en particulier, ainsi que des lésions cardiaques et de la bradycardie, les enquêtes sur la fréquence insolite des cas de mort subite, les examens de plus de 600 enfants habitant dans des chaumières à triatomes, n'avaient fourni aucun résultat positif.

Or, à peu près simultanément, la virulence des parasites des réduvidés vient d'être établie au Mexique et à Paris. D'une part, Mazzotti, en utilisant le xénodiagnostic, a pu mettre en évidence deux cas, aux mines d'El Carmen, dans l'Etat d'Oaxaca où le vecteur est Rhodnius prolixus; d'autre part, nous observions à Paris un cas accidentel survenu chez une de nos collaboratrices (Mlle A. Herr) qui reçut dans l'œil droit, le 30 novembre 1938 , le contenu intes- 
tinal d'exemplaires de Triatoma pallidipennis récoltés dans des terriers de Neotoma (Hodomys) alleni, à quelques kilomètres des habitations les plus proches. Ce dernier cas est particulièrement intéressant, car il montre l'adaptation immédiate à l'homme d'un virus qui depuis des millénaires passait des triatomes aux rongeurs et de ces derniers aux triatomes.

Nous croyons utile de donner un résumé de ces diverses observations :

\section{Gas naturels observés à la mine d'El Garmen (Oaxaca)}

(L. Mazzotti)

a) Rufina Grijalva (pI. XXI, fig. 1). - Femme métis âgée de 30 ans, arrivée à El Carmen depuis six mois et habitant une chaumière dont le toit et les parois de paille hébergeaient de nombreux Rhodnius prolixus.

Deux mois avant notre visite, cette malade a présenté de l'œdème palpébral, surtout accusé à droite, puis étendu ensuite à gauche, non accompagné de fièvre. Elle a encore un œedème visible, surtout du côté droit; pas d'adénite, pouls à 90 .

Formule leucocytaire.

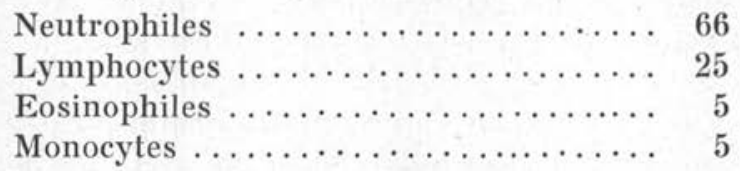

Xénodiagnostic. - Le 2 novembre 1938, 9 larves neuves de Triatoma phyllosoma piquent la malade; cinquante jours plus tard, à la dissection, 7 sont positives.

Diagnostic direct. - Sur cinq gouttes épaisses examinées, une seule présente un trypanosome.

Pronostic. - Il s'agit en somme d'un cas de maladie de Chagas à allure bénigne.

b) Agripina Montes. - Femme âgée de 40 ans, habitant El Carmen depuis deux ans. Un mois avant notre passage, cette malade a présenté de l'œdème palpébral gauche. On observe actuellement, à l'angle interne de l'œil gauche, un petit nodule semblant être une dacryocystite en voie de régression. Pas d'adénites; pouls à 80 . Aucune lésion cardiaque. Foie et rate normaux.

Formule leucocytaire.

Neutrophiles $\ldots \ldots \ldots \ldots \ldots \ldots \ldots \ldots .66$

Lymphocytes ................. 24 
Eosinophiles .................. 5

Monocytes .................. 5

Xénodiagnostic. - Sur cinq larves de Triatoma phyllosoma ayant piqué cette malade en novembre 1938, quatre se montrent infectées à la dissection cinquante jours plus tard.

Diagnostic direct. - Négatif après examen de quatre gouttes épaisses.

Pronostic. - Ici encore, il s'agit d'un cas typique et bénin de maladie de Chagas. D'ailleurs, dans ce même village où un certain nombre d'habitants ont présenté de l'œedème oculaire et facial, persistant pendant plusieurs semaines, quelques-uns seulement ont eu des accès fébriles de courte durée. Il est vrai que ces gens, habitués à de durs travaux, ne font guère attention aux légers symptômes qu'ils peuvent présenter et les attribuent le plus souvent à la fatigue et non à la fièvre.

2. Gas accidentel de laboratoire (A. Herr et L.-C. Brumpt)

Mlle A. Herr, Docteur en Médecine, aide technique du service de la Recherche scientifique, en utilisant pour une injection une aiguille bouchée accidentellement, reçoit dans l'œil droit, le 30 novembre 1938, à Paris, une partie du contenu de sa seringue, renfermant des déjections de Triatoma pallidipennis, récoltés dans un terrier de rongeur [Neotoma (Hodomys) alleni], aux environs de la ville de Colima (Mexique).

En dépit d'une désinfection immédiate à l'alcool, Mlle A. Herr ressent, le 13 décembre 1938, alors qu'elle se trouvait en parfaite santé, une douleur à l'œil droit, à l'angle interne duquel elle remarqua une rougeur. Le 14 décembre, elle constata un œedème palpébral et sous-palpébral, accompagné de larmoiement. Un spécialiste consulté diagnostiqua une dacryocystite légère. En outre, la malade éprouve une sensation de malaise général, de la courbature lombaire, et présente de petites adénites prétragienne et sous-maxillaire droite, douloureuses à la pression; température $37^{\circ} 5$.

Malgré des examens de sang négatifs, nous demandons télégraphiquement en Argentine un médicament actif au $\mathrm{D}^{r}$ Mazza, et ayant appris que ce produit venait de chez Bayer, nous obtenons, grâce à l'aimable intervention du Professeur Kikuth, un certain nombre d'ampoules de ce médicament expédiées par M. Kœnig, Directeur de ces laboratoires. Le 16 décembre, à la suite d'un traitement local, la dacryocystite a presque totalement disparu et le canal lacrymal est redevenu perméable. Cependant, l'œè̀me pal- 
pébral persiste, s'étend et la malade souffre de myalgies dans la nuque et les lombes, ainsi que de céphalée ; le soir, la température est de $38^{\circ} 5$. Les examens de sang sont encore négatifs à frais et, après coloration, en goutte épaisse.

Le 18 décembre, l’œè̀me subit une poussée inflammatoire, rougit et semble limité par un bourrelet. Bien qu'il ne s'agisse pas d'un érysipèle typique, comme les examens de sang sont encore négatifs, la malade prend des sulfamidés. Le 21 décembre, on interrompt l'administration de ces médicaments dont l'action a

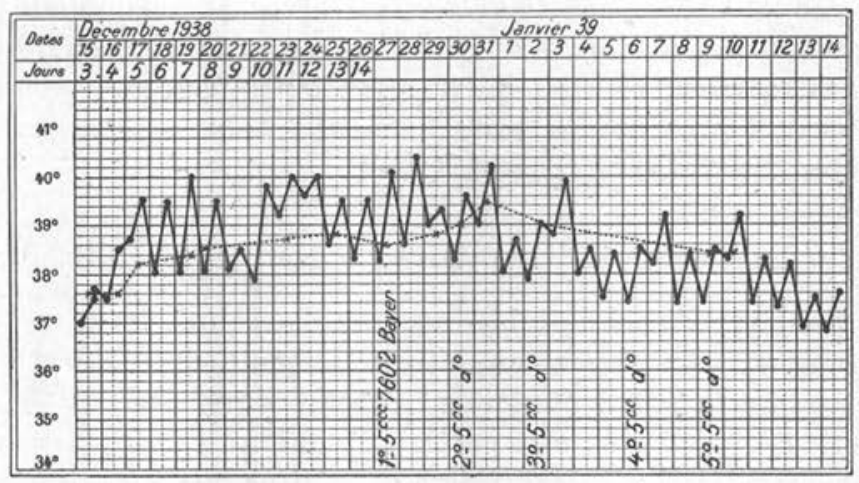

Fig. - Courbe fébrile d'un cas accidentel de laboratoire ayant débuté par une contamination de l'œil droit. D'après A. Herr et L.-C. Brumpt (1939).

été nulle. La formule sanguine fait apparaitre de la monocytose. Du 22 au 27 décembre, des signes nouveaux se manifestent: des ganglions carotidiens et un ganglion axillaire, gros comme un pois. Bien que la fièvre atteigne $40^{\circ}$ avec légère rémission matutinale (fig.), l'état général n'a rien d'alarmant; la malade aime à recevoir des visites; elle peut lire et s'alimente assez normalement.

L'examen physique montre que l’œè̀me palpébral, qui a perdu son aspect inflammatoire et présente une légère desquamation furfuracée, s'est étendu sur la joue droite et la paupière supérieure gauche. Il s'agit d'une inflammation dermique, non douloureuse à la pression et ne laissant pas de godet. La malade ne présente ni conjonctivite, ni dacryoadénite.

La rate, non palpable, est nettement percutable sur quatre travers de doigt; le foie est de volume normal; la langue est blanche, la gorge normale et les réflexes également normaux. Le 
pouls régulier et bien frappé est légèrement dissocié; il ne dépasse jamais 100, même quand la température atteint $40^{\circ}$. Les bruits du cœur sont normaux et la tension reste constamment entre 11 et 6 au Vaquez. Les urines sont peu abondantes; elles ne contiennent ni sucre, ni albumine.

La formule sanguine montre toujours une leucocytose subnormale avec mononucléose.

En dépit de nombreux examens, de sang négatifs, les symptômes cliniques et l'accident qui avait marqué le début de la maladie permettent de poser le diagnostic de maladie de Chagas. Toutefois, avant de traiter la malade par le médicament 7.602 "Bayer », il est procédé à un xénodiagnostic. A cet effet, 13 exemplaires de Rhodnius prolixus et de Triatoma infestans (nymphes au $4^{\circ}$ stade) piquent la malade sans que celle-ci éprouve la moindre douleur; c'est seulement quinze jours plus tard que les piqûres sont le siège d'un érythème maculopapuleux, doux au toucher et légèrement prurigineux.

Lé xénodiagnostic devient positif le $37^{\circ}$ jour ; de nombreux trypanosomes métacycliques se trouvant dans les déjections des réduvidés, plusieurs souris sont infectées et ce virus est actuellement inscrit à l'Institut de Parasitologie sous le nom de «CruziHerr».

\section{RÉSUMÉ}

1. Le Trypanosoma cruzi, signalé au Mexique dans le tube digestif de divers Réduvidés: T. barberi, $T$. dimidiata, T. pallidipennis, T. phyllosoma, T. rubida, Rhodnius prolixus, peut être pathogène pour l'homme, ainsi qu'il résulte de deux cas cliniques observés au Mexique et confirmés par le xénodiagnostic, et d'un cas accidentel survenu à Paris, chez une assistante de l'Institut de Parasitologie.

2. Il existe au Mexique 11 espèces de triatomes et une espèce de Rhodnius. Les seules espèces étudiées dans ce travail sont les plus communes; elles se rencontrent dans les terres chaudes. Ce sont : T. barberi, T. dimidiata, T. pallidipennis, T. phyllosoma à ailes longues, ailes moyennes et ailes courtes, T. rubida, enfin Rhodnius prolixus qui présentent parfois un pourcentage d'infection élevé par Trypanosoma cruzi.

3. Les seuls animaux réservoirs de virus actuellement connus au Mexique sont le chien et un tatou (Dasypus novemcinctus mexicanus). Un rongeur, Neotoma (Hodomys) alleni, dont les 
terriers renferment de nombreux Triatoma pallidipennis infectés, est certainement un animal réservoir de virus important. Il doit en exister beaucoup d'autres dont la détermination reste à établir.

\section{EXPLICATION DES PLANCHES XXI-XXV}

\section{PlaNehe XXI}

Fig. 1. - Une malade d'El Carmen, Rufina Grijalva, dont l'infection a été établie par le xénodiagnostic.

Fıg. 2, - Enquête épidémiologique. Trone d'un gros Ficus sp. habité par de nombreux Neotoma (Hodomys) alleri, qui déposent leurs déjections dans des amas marqués par une + . Un chasseur, indiqué par une flèche, qui s'est souvent abrité la nuit entre les racines où il se trouve, $\mathrm{y}$ a souvent été piqué par des Triatomes, hôtes habituels des terriers de Neotoma.

\section{Planche XXII}

Fı́. 3. - Dans la région de Colima (Mexique), une espèce de triatome, T, pallidipennis se rencontre dans la nature, loin des habitations humaines, sous les racines de grands arbres où nichent des tatous et divers 1ongeurs, en particulier un gros rat, Neotoma (Hodomys) alleni.

Fig. 4. - Habitat des triatomes. L'examen de plusieurs nids de « calandria 》 suspendus aux arbres n'a pas permis de trouver au Mexique le Triatoma arthuri qui s'y rencontre parfois en abondance au Venezuela et dans quelques autres pays de l'Amérique latine.

\section{Planche XXIII}

Fig. 5. - Les cactées candélabre (flèche blanche) du Mexique sont parfois creusés de nombreux nids de pies (flèche noire). Aucun triatome ne se trouvait dans ceux qui ont été examinés et qui renfermaient des scorpions qui les avaient peut-être détruits.

Fig. 6. - Habitat des triatomes ( $T$. phyllosoma). Dans la région de Tehuantepec (Mexique), ces insectes se rencontrent facilement dans les tas de tuiles de réserve où ils vivent en compagnie de nombreux scorpions qui, probablement, les détruisent.

\section{Planche XXIV}

Fig. 7. - Le tatou (Dasypus novemcinctus) est un important réservoir de Trypanosoma cruzi dans toute l'Amérique latine. Cliché pris aux environs de Colima (Mexique).

Fig. 8. - Un chasseur de Colima, Felipe Garcia, nous apporte divers animaux; il tient dans sa main gauche trois Neotoma (Hodomys) alleni.

\section{Planche XXV}

Fig. 9. - Mode de couchage des paysans de la région de Tehuantepec (Mexique). Dans les nattes et dans les parois crevassées des murs, de nombreux Triatoma phyllosoma infectés par $T$. cruzi ont été récoltés.

Fıg. 10. - Habitants d'une maison à Triatoma phyllosoma de Tehuantepec (Mexique). Aucun d'eux ne présentait de signes cliniques de la maladie de Chagas. 
ANNALES DE PARASITOLOGIE

Planche XXI

T. XVII, No 4, 1er Juillet 1939 (Mémoire E. Brumpt, Mazzotri et L. C. Brcmpt)

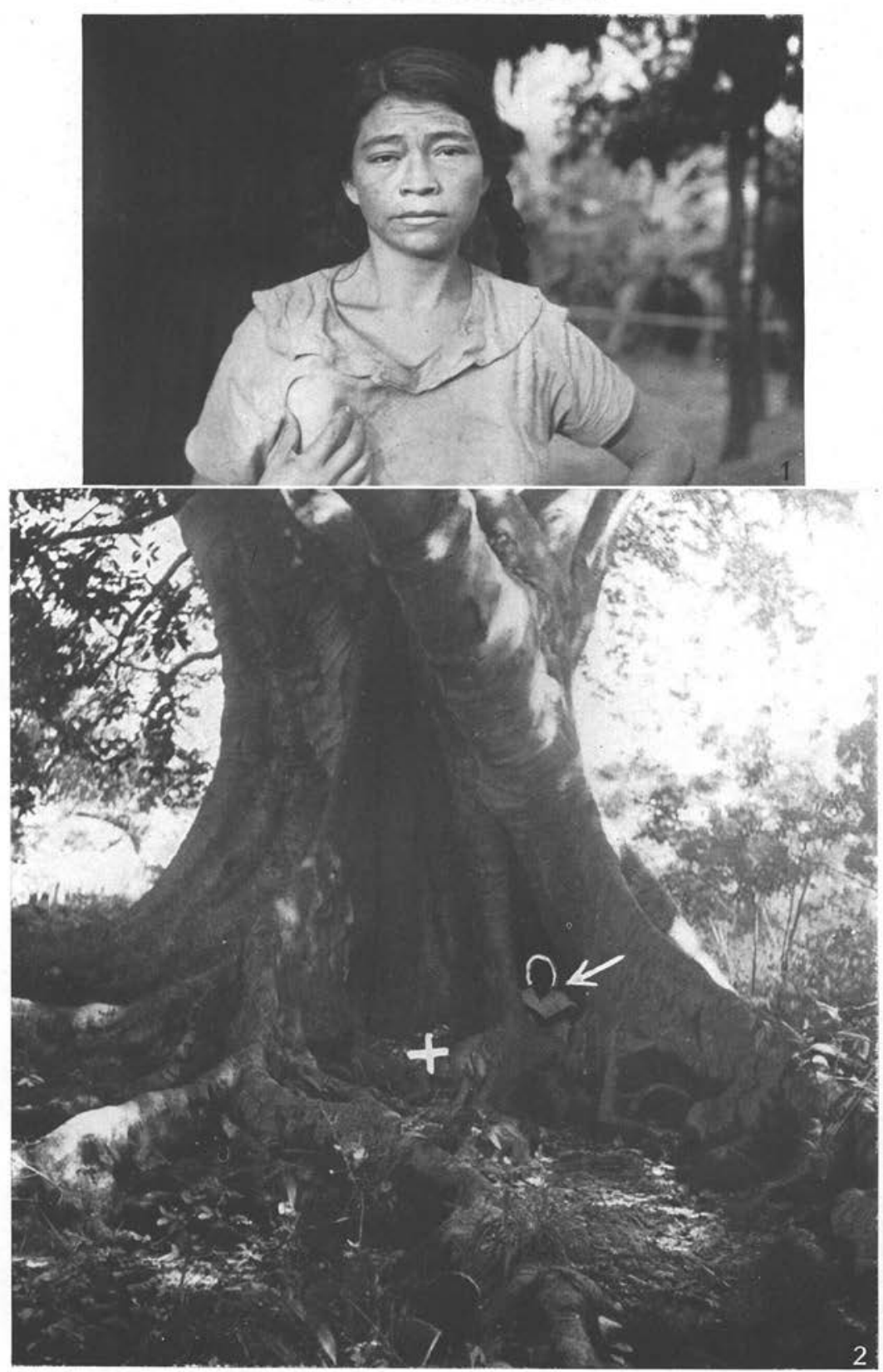



T. XVIl, No 4, 1er Juillet 1939 (Mémoire E. Brumpt, Mazzotti et L. C. Brumpt)
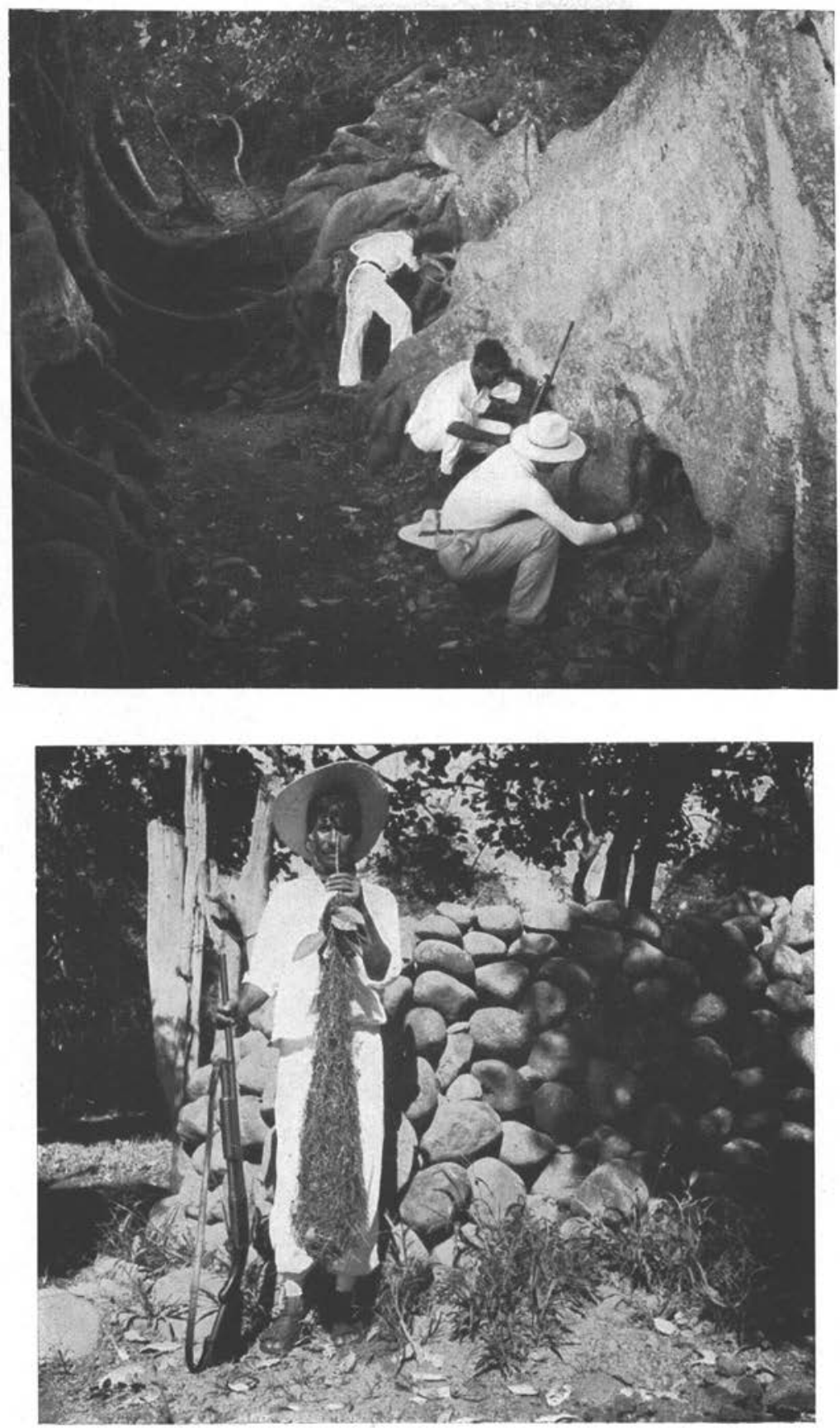
T. XVII, No 4, 1er Juillet 1939 (Mémoire E. Brumpt, Mazzotti et L. C. Brumpt)

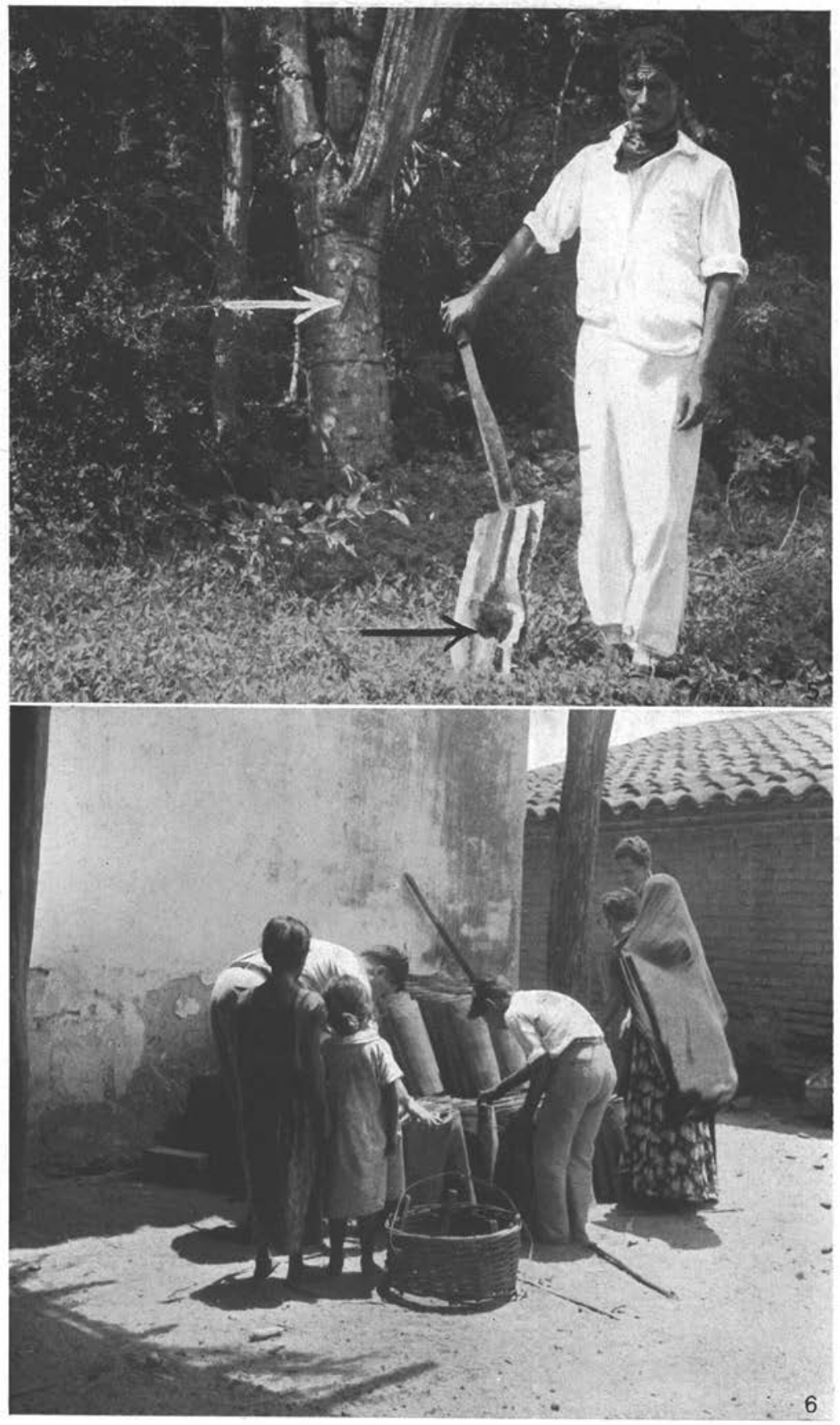

Masson et Cie, Editeurs 

T. XVII, No 4, 1er Juillet 1939 (Mémoire E. Brumpt, Mazzott et L. C. Brumpt)
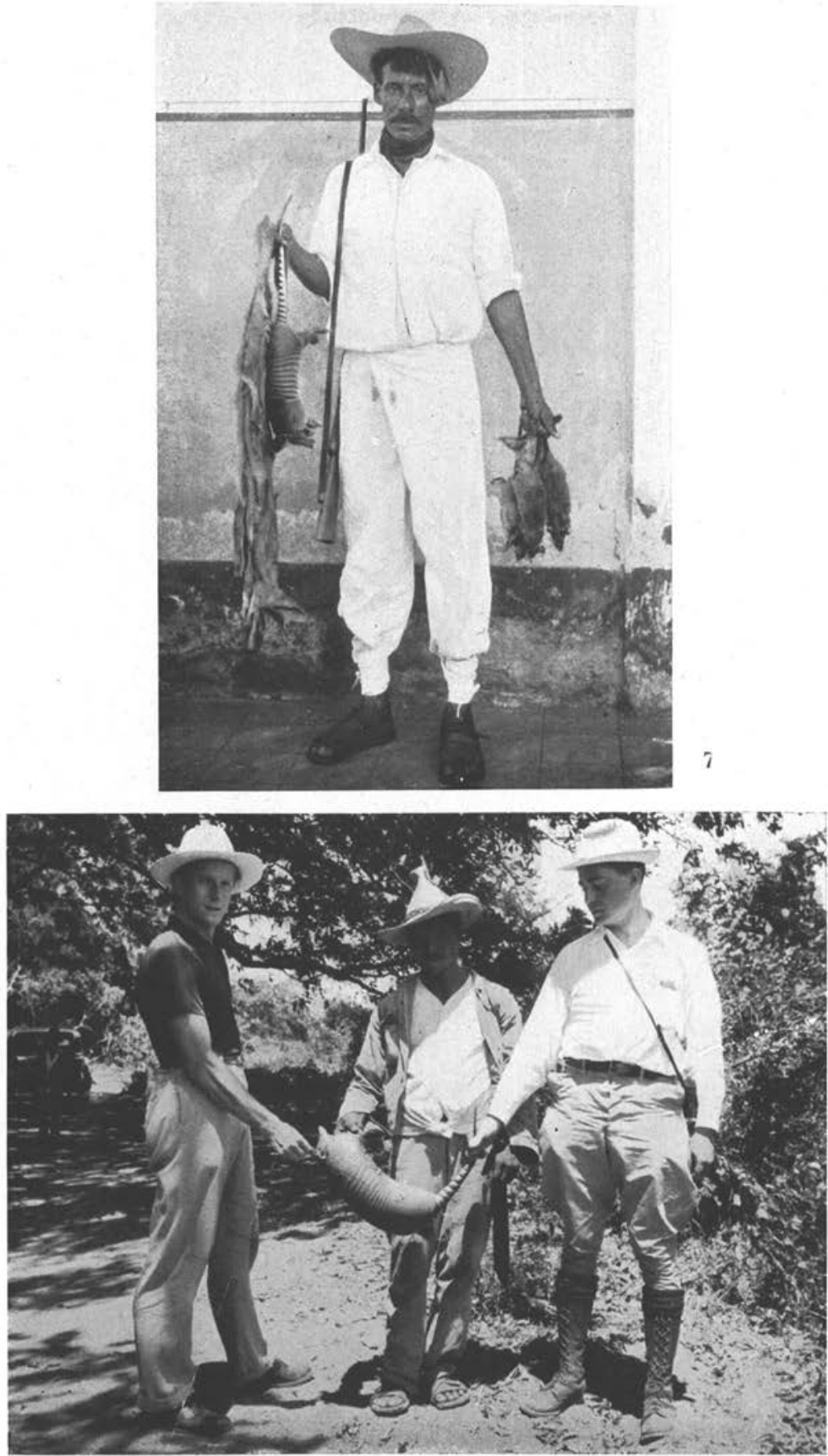

T. XVII, No 4, 1er Juillet 1939. (Mémoire E Brumpt, Mazzoti et L.C. Brumpt)
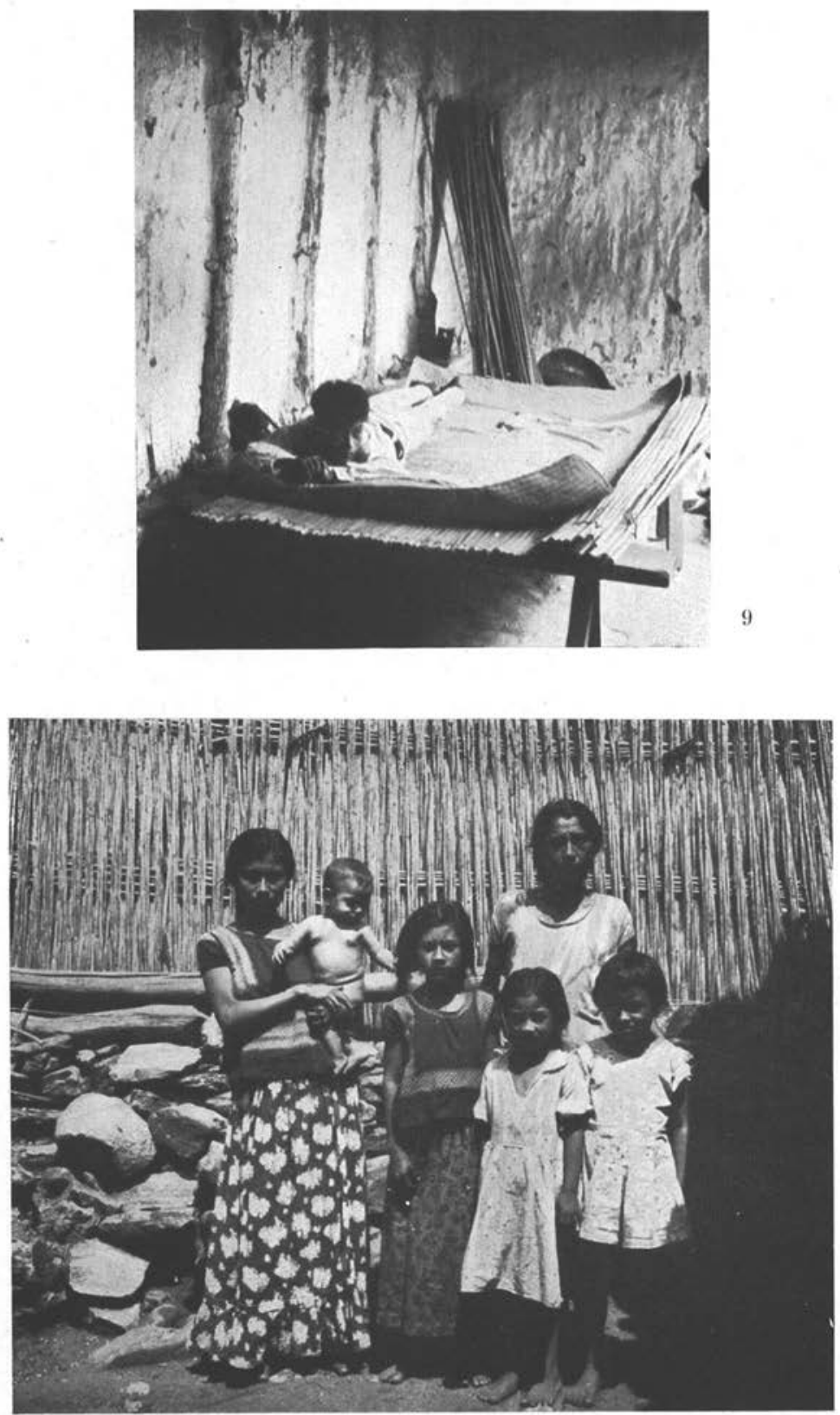

4. A en juger par les cas de maladie de Chagas signalés dans ce travail, l’infection mexicaine paraît bénigne, et, jusqu'à ce jour, aucune lésion cardiaque, ni aucune mort subite qui lui soit imputable, ne nous ont été signalées au cours de nos enquêtes. Ces observations n'ont rien d'insolite, car les souches de Trypanosoma cruzi, étudiées dans les divers pays de l'Amérique latine, présentent des degrés de virulence très variables dans les conditions expérimentales, et vraisemblablement aussi dans la nature.

\section{Bibliographie}

Bernal Flandes. - Anotaciones sobre triatomas y trypanosomas. Rev. med. veracruzana, XVIII, mai 1938 , p. 2533.

Brumpt (E.). - Le Trypanosoma cruzi évolue chez Conorhinus megistus, Cimex lectularius, Cimex boueti et Ornithodorus moubata. Cycle évolutif de ce parasite. Bull. Soc. Path. Exot., V, 1912, 360.

Evolution de Trypanosoma lewisi, nabiasi, blanchardi chez les puces et les punaises. Transmission par les déjections. Comparaison avec T. cruzi. Bull. Soc. path. exot., VI, 1913, 167.

Le xénodiagnostic. Applications au diagnostic de quelques affections. parasitaires et en particulier à la trypanosomose de C. Chagas. Bull. Soc. Path. Exot., VII, 1914, 706.

Faits expérimentaux et cliniques concernant le mode de transmission de la maladie de Chagas ou trypanosomose américaine. C.R. Soc. Biol., CXXX, mars 1939, p. 1197 .

La maladie de C. Chagas. Exemple d'infection très répandue, considérée jusqu'à ce jour comme rare faute d'enquêtes épidémiologiques méthodiques. Presse Médicale, n 50, 24 juin 1939, p. 1013.

Quelques faits épidémiologiques concernant la maladie de C. Chagas. Presse Médicale, $\mathrm{n}^{\circ}$ 54, 8 juillet 1939.

Castro Ferreira (L. De) et Deane (L.). - Novo depositorio sylvestre do Schizotrypanum cruzi (Chagas 1909) à Irara, Tayra barbara (L.). Brasil medico, LII, 1938, p. 1159.

Herr (Annette) et Brumpt (L.-C.). - Un cas aigu de maladie de Chagas contracté accidentellement au contact de triatomes mexicains. Observation et courbe fébrile. Bull. Soc. Path. Exot., XXXII, 1939, p. 565.

Hoare (C. A.). - Miscellanea protozoologica. II. Développement of mammalian trypanosomes in the body-cavity of caterpillars. Trans. Roy. Soc. Trop. med., XXXII, 1938, p. 8.

Kofoid (C.-A.) et WhiтAker (B. G.). - Natural infection of american human trypanosomiasis in 2 species of cone-nosed bugs, Triatoma protracta Uhler and Triatoma uhleri Neiva, in Western United States. Journ. Parasitol., XXII, 1936, p. 259.

Mazza (S.). - A propos du procès-verbal (note présentée par Marchoux). Bull. Soc. Path. Exot., XXXII, 10 mai 1939.

Mazzotti (L.). - Investigacion sobre la existencia de la enfermedad de Chagas en el pais. Demostracion de tripanosomas en los reduvideos trasmisores. Medicina, Mexico, XVI, 584-585, déc., 25, 1936. 
Mazzotri (L.). - Infeccion natural de Trypanosoma cruzi de Chagas en Triatoma phyllosoma, Burmeister y T. pallidipennis de la Costa del Pacifico de Mexico. Medicina, Mexico, XVII, 1937, p. 161-166.

- Infeccion natural de Trypanosoma cruzi de Chagas en Triatoma dimidiata (Latreille) de los estados de Yucatan, Campeche, Chiapas, Vera Cruz y Jalisco. Medicina, Mexico, XVII, 1937, p. 283-286.

- I. Presencia de Rhodnius prolixus Stal en Mexico. II. Trypanosoma cruzi en un Armadillo de Colima. Medicina, Mexico, XVIII, 1938, p. 606.

- Dos casos de enfermedad de Chagas en el Estado de Oaxaca. Note présentée le 2 janvier 1939 à l'Académie de Médecine de Mexico.

- Datos para la investigacion de la enfermedad de Chagas. Boletin epidemiologico, II, $\mathrm{n}^{\circ} 10-11,1939$, p. 303.

Rodhain (J.). - Note sur Trypanosoma minasense Chagas. C.R. Soc. Biol., CXXV, 1937, p. 1034 .

- Notes sur Trypanosoma minasense Chagas. Evolution du trypanosome du Saimiri chez divers arthropodes. C.R. Soc. biol., CXXVI, 1937, p. 69.

Rodhain (J.) et Brutsaert (P.). - L'évolution des Trypanosoma lewisi et Trypanosoma cruzi chez Melophagus ovinus, C.R. Soc. biol., CXVIII, 1935, p. 1228.

Tejera (E.) - Un nouveau flagellẻ de Rhodnius prolixus, Trypanosoma (ou Crithidia) rangeli n. sp. Bull. Soc. Path. Exot., XIII, 1920, p. 527.

Torrealba (J.F.). - Mas investigaciones para el estudia de la Enfermedad de Chagas en el districto Zarara. Medicatura de sanidad de Zarara (Guarico, Venezuela). In- $8^{\circ}$ de 45 pages, 1937.

Institut de Parasitologie de la Faculté de médecine de Paris (Directeur: Professeur E. Brumpt) et Instituto de Salubridad y Enfermedades

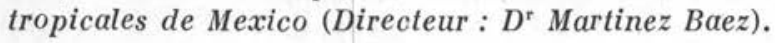

